\title{
Universidades socialmente responsables
}

\author{
Socially responsible universities
}

\author{
Patricia Kent \\ ARGENTINA
}

Resumen: A partir de la necesaria reflexión sobre un tema que se presenta hoy como central para la vida universitaria, el artículo aspira a inducir el intercambio y debate de ideas entre las instituciones de educación superior latinoamericanas. En la introducción se comparten algunas aproximaciones teóricas a partir de la revisión bibliográfica y la experiencia profesional. A continuación, se presentan como caso testigo dos investigaciones desarrolladas en una universidad argentina, cuyo modelo de abordaje puede ser replicado en casas de estudios colegas dispuestas a intentarlo. Para finalizar se plantean algunos aspectos a considerar ante el desafío que implica asumir la responsabilidad social universitaria como mecanismo para brindar las respuestas que la sociedad demanda.

Palabras clave: educación superior, gestión universitaria, responsabilidad social universitaria.

Abstract: Based on the necessary reflection on a topic that is presented today as central to university life, the article aims to induce the exchange and debate of ideas among Latin American higher education institutions. In the introduction some theoretical approaches are shared based on the bibliographic review and professional experience. Next, two investigations, carried out in an Argentine university, whose approach model can be replicated in colleges willing to try, are presented as a witness case. To finish, some aspects to consider in the face of the challenge of assuming university social responsibility as a mechanism to provide the responses that society demands

Keywords: higher education, university management, university social responsibility.

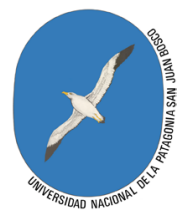

\footnotetext{
Patricia Kent es Magister y Doctora en Desarrollo Humano y Sustentable por la Universidad Bolivariana de Chile, Master en Ecoauditorías y Planificación Empresarial del Medio Ambiente, del Instituto de Investigaciones Ecológicas (España), y Licenciada en Administración de la Universidad Nacional de la Patagonia. Actualmente es docente de postgrado y Directora regular del Instituto de Responsabilidad Social para el Desarrollo Sustentable -Getace- de la Universidad Nacional de la Patagonia, en Trelew, Argentina. 


\title{
Universidades socialmente responsáveis
}

\begin{abstract}
Resumo: Com base na reflexão necessária sobre um tema hoje apresentado como central da vida universitária, o artigo visa induzir ao intercâmbio e ao debate de ideias entre instituições de ensino superior latino-americanas. Na introdução, algumas abordagens teóricas são compartilhadas com base na revisão bibliográfica e na experiência profissional. Em seguida, duas investigações realizadas em uma universidade argentina, cujo modelo de abordagem pode ser replicado em outras faculdades dispostas a tentar, são apresentadas como um caso de testemunha. Para finalizar, alguns aspectos a serem considerados à luz do desafio de assumir a responsabilidade social da universidade como um mecanismo para fornecer as respostas que a sociedade exige.
\end{abstract}

Palavras-chave: educação superior, gestão universitária, responsabilidade social universitária.

\section{Aportes para la reflexión}

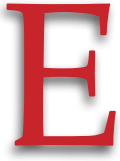

I artículo propone una reflexión sobre los modos de gestión implementados en las instituciones de educación superior latinoamericanas en un marco de responsabilidad social universitaria (RSU).

En ese orden, Peter Drucker, uno de los referentes mundiales en temas de management, afirmaba en uno de sus libros"las viejas formas de dirección de empresas no prestaron atención a las personas ni a los valores, por el contrario, los han subestimado y anulado, de esa manera han debilitado las posibilidades de desarrollo atentando contra sus propios objetivos económicos". (Cleri, 2007, p.187)

Parafraseando respetuosamente al autor, es posible afirmar que las formas de gestión universitaria actuales, en varias instituciones de educación superior latinoamericanas, no prestan atención a las personas ni a los valores, por el contrario, los subestiman y frecuentemente los anulan, de esa manera debilitan sus posibilidades de desarrollo y atentan contra sus propios objetivos constitutivos.

Prestar atención a las personas y a los valores desde la universidad, induce a asumir que por su identidad está llamada a formar profesionales de excelencia, imbuidos de principios que guíen su comportamiento al servicio de sus semejantes, como contribución al logro de una sociedad más justa, más pacífica y más humana, lo cual se concretará en la medida en que se adopte como un proceso continuo y permanente, el ejercicio de repensar la misión universitaria en función de su responsabilidad social. 
"Ser socialmente responsable impone a las personas, como miembros de la sociedad, respetar las obligaciones legales, demostrar su sensibilidad social (capacidad para reconocer y responder a las demandas sociales), y cumplir con sus deberes éticos. La ética es la génesis de la responsabilidad social" (Kent, 2020, p.88) y en este sentido, Schvarstein sostiene que "el ejercicio de la responsabilidad social mitiga y seguirá mitigando la insatisfacción de las necesidades sociales. Es nuestro compromiso social lo que está en juego y ello remite al terreno de la ética" (2003, p.256).

Asumir la responsabilidad social, como una forma de desarrollo humano diferente, exhorta a la universidad a consolidar su capacidad para producir un profundo cambio en su cultura organizacional basado en valores, entre ellos coherencia, compromiso, confianza, convicción, equidad, ética, honestidad, inclusión, pertenencia, solidaridad y transparencia, para lograr modificaciones sólidas y perdurables en los comportamientos individuales y colectivos de sus claustros, a partir de procesos que se construyen desde la sensibilización, la concienciación y la capacitación.

Dice Calderón (2016, p.200) que "la responsabilidad social universitaria será posible, cuando sus miembros la asuman como parte de una cultura en la cual el discurso y la acción, se conjuguen para promover el cambio social, el desarrollo y transformación del entorno", de modo tal que el ejercicio de su poder transformador de la sociedad debe conducir a las instituciones de educación superior a redefinir sus funciones sustanciales para hacer posible un desarrollo humano responsable y sostenible.

La RSU se asume como una propuesta de dinámica organizacional tendiente a encontrar equilibrio y conectividad entre los actores involucrados, procurando acciones hacia el entorno social y ambiental desde una iniciativa autónoma, de modo tal que su misión trascienda sus obligaciones legales, con el propósito de cimentar una nueva cultura universitaria.

Al coincidir con Torres Pernalete y Trápaga Ortega (2010, p.186) cuando afirman "este paradigma de universidad socialmente responsable va a generar un tipo de profesional diferente, un egresado capaz de dar respuesta a los problemas del mundo de hoy, con claro sentido de ciudadanía global, un profesional responsable por su participación no solo en su entorno familiar, profesional y social, sino un egresado que pueda convertirse en un agente de cambio local, nacional, regional o internacional, que genere aprendizajes permanentes", es válido pensar la RSU como un proceso que reconoce el papel central de la universidad en 


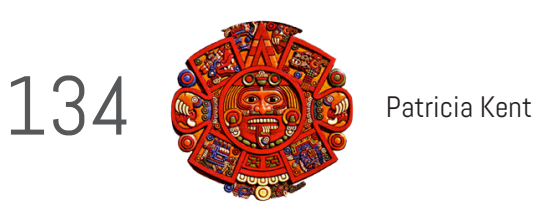

orden a fortalecer el compromiso social, ético y político de la educación superior, que conlleva la necesidad de consolidar su rol institucional protagónico en la transformación educativa, formando estudiantes que interpreten su futura profesión como una posibilidad de servicio de cara a las demandas sociales en términos de una ciudadanía responsable.

En el Proyecto Universidad: Construye País (2001-2006, p.4), los investigadores sostienen que "Por misión, las universidades están dedicadas a crear conocimiento y formar científicos, humanistas y profesionales orientados a satisfacer las necesidades de desarrollo del país. Los temas como la pobreza, la desintegración social, el desarrollo del capital social y la protección de los recursos naturales (desarrollo sustentable) deberían estar en el centro de sus preocupaciones". Con esta visión, las instituciones de educación superior deben influir en la toma de conciencia sobre la problemática socioambiental de su entorno, con alternativas que viabilicen una formación profesional comprometida con el bien común.

Los problemas sociales y ambientales atraviesan transversalmente a la sociedad y lamentablemente son compartidos por todos los habitantes del planeta; por lo tanto, es importante advertir que unilateralmente ningún sector podrá enfrentarlos con éxito. Asimismo, se debe reconocer el lugar a ocupar por las universidades adquiere relevancia en términos de su contribución al trabajo requerido para la construcción de acuerdos y alianzas tendientes al diseño de modelos basados en el fortalecimiento del capital social, de aplicación no solo en las agendas sino más precisamente en las decisiones y acciones para incorporarlos desde la gestión, la docencia, la extensión y la investigación, en la formación de los futuros graduados.

Al respecto cabe mencionar los Objetivos de Desarrollo Sostenible definidos en apoyo de la Agenda 2030 de la Organización de Naciones Unidas (Naciones Unidas, 2015), cuya preocupación y ocupación central implica el compromiso de todos los actores sociales, públicos, privados y del tercer sector, con la construcción de sociedades equitativas y respetuosas del medio ambiente.

En este caso, el objetivo 4 propone: "garantizar una educación inclusiva para todos y promover oportunidades de aprendizaje duraderas que sean de calidad y equitativas", de modo tal que al desarrollar su tarea sustantiva, las universidades deben implementar estrategias y procedimientos que promuevan una educación de calidad desde la formación, con la firme convicción de que se trata de uno de los motores más poderosos y probados para garantizar la movilidad social ascendente y el desarrollo sostenible.

Para el cumplimiento del objetivo 4, la ONU ha establecido un conjunto de metas con vistas a 2030 entre ellas algunas vinculadas directamente con la educación universitaria: 
- "Para 2030, asegurar el acceso en condiciones de igualdad para todos los hombres y las mujeres a una formación técnica, profesional y superior de calidad, incluida la enseñanza universitaria".

- "Para 2030, aumentar considerablemente el número de jóvenes y adultos que tienen las competencias necesarias, en particular técnicas y profesionales, para acceder al empleo, el trabajo decente y el emprendimiento".

- "Para 2030, garantizar que todos los alumnos adquieran los conocimientos teóricos y prácticos necesarios para promover el desarrollo sostenible, entre otras cosas mediante la educación para el desarrollo sostenible y la adopción de estilos de vida sostenibles, los derechos humanos, la igualdad entre los géneros, la promoción de una cultura de paz y no violencia, la ciudadanía mundial y la valoración de la diversidad cultural y de la contribución de la cultura al desarrollo sostenible".

Asimismo, en Argentina la ley nacional de educación superior ${ }^{1}$ establece en su artículo $3^{\circ}$ :

- "La Educación Superior tiene por finalidad proporcionar formación científica, profesional, humanística y técnica en el más alto nivel, contribuir a la preservación de la cultura nacional, promover la generación y desarrollo del conocimiento en todas sus formas, y desarrollar las actitudes y valores que requiere la formación de personas responsables, con conciencia ética y solidaria, reflexivas, críticas, capaces de mejorar la calidad de vida, consolidar el respeto al medio ambiente, a las instituciones de la República y a la vigencia del orden democrático.

Mientras que, en el artículo $4^{\circ}$ se puntualizan los objetivos de la educación superior en los siguientes términos:

- a) Formar científicos, profesionales y técnicos, que se caractericen por la solidez de su formación y por su compromiso con la sociedad de la que forman parte;

- b) Preparar para el ejercicio de la docencia en todos los niveles y modalidades del sistema educativo;

- c) Promover el desarrollo de la investigación y las creaciones artísticas, contribuyendo al desarrollo científico, tecnológico y cultural de la Nación;

- d) Garantizar crecientes niveles de calidad y excelencia en todas las opciones institucionales del sistema;

${ }^{1}$ Véase: Argentina. Ley Nacional N² 24521.

http://servicios.infoleg.gob.ar/infoleglnternet/anexos/25000-29999/25394/texact.htm 
- e) Profundizar los procesos de democratización en la Educación Superior, contribuir a la distribución equitativa del conocimiento y asegurar la igualdad de oportunidades;

- f) Articular la oferta educativa de los diferentes tipos de instituciones que la integran;

- g) Promover una adecuada diversificación de los estudios de nivel superior, que atienda tanto las expectativas y demandas de la población como a los requerimientos del sistema cultural y de la estructura productiva;

- h) Propender a un aprovechamiento integral de los recursos humanos y materiales asignados;

- i) Incrementar y diversificar las oportunidades de actualización, perfeccionamiento y reconversión para los integrantes del sistema y para sus egresados;

- j) Promover mecanismos asociativos para la resolución de los problemas nacionales, regionales, continentales y mundiales.

De modo tal que las universidades deben ocupar el lugar que les corresponde, en el contexto del sistema de educación superior, para la implementación de políticas tendientes a implementar los cambios requeridos para el logro de los objetivos enunciados

Contribuir con la búsqueda de respuestas a las necesidades de la sociedad requiere de las instituciones de educación superior articular sus funciones sustanciales de docencia, extensión e investigación, con una mirada integral que garantice prestar la ineludible atención a las consecuencias de su accionar sobre el entorno social y ambiental, interno y externo, apoyadas en una gestión institucional socialmente responsable.

Es preciso afianzar el compromiso con la RSU estableciendo políticas de gestión responsables y sostenibles, así como los mecanismos específicos que faciliten su instrumentación, considerando entre otros aspectos: asignaciones presupuestarias suficientes para garantizar igualdad de oportunidades de acceso y permanencia mediante el otorgamiento de becas y estímulos a los estudiantes, cuerpos docentes-investigadores de calidad y equipos de apoyo eficientes, así como también, reformas en las estructuras funcionales e inversiones en infraestructura y tecnología.

La universidad debe revisaryadecuar de manera permanente sus propuestas curriculares y apelar a la formación, capacitación y perfeccionamiento continuos de sus equipos docentes y técnicos, dotándolos de los recursos necesarios para diseñar e implementar reformas que contemplen la diversidad, complejidad, transversalidad y transdisciplinariedad de los contenidos requeridos para apropiarse del paradigma de la responsabilidad 
social, ya no como alternativa sino como requerimiento, de manera que estén preparados para compartir su experiencia académica y profesional con sus estudiantes, afianzando sus capacidades y potencialidades.

Asimismo, resulta imperioso abandonar la concepción fragmentada asociada a la idea tradicional de extensión universitaria como actividad para dar respuestas a las necesidades de capacitación o actualización disciplinar extracurricular, arraigada y practicada en muchas instituciones de educación superior, cuando en realidad, se trata de una función básica dearticulación, para vincular los desarrollos académicos y de investigación, en beneficio de la sociedad. La extensión debe ser comprendida en términos de servicio para la promoción del ejercicio de una ciudadanía responsable, de modo tal que sus egresados estén capacitados para desarrollar su práctica profesional con compromiso comunitario.

Por último, se requiere unificar concepciones teóricas, enfoques metodológicos y criterios prácticos que, desde la investigación universitaria, permitan hacer un abordaje interdisciplinar de la RSU. Los impactos humanos, éticos e institucionales producto de esa tarea deben lograr una realimentación positiva con la sociedad, contemplando las demandas y requerimientos de los actores sociales, internos y externos de su entorno mediato e inmediato. No se trata de una función alejada de la formación, por el contrario, es la base de la producción del conocimiento y del desarrollo tecnológico, que debe estar incorporada a las prácticas universitarias para garantizar el contacto de los estudiantes desde el inicio de la carrera con la realidad, la complejidad y la diversidad en las cuales se insertarán profesionalmente, y para que puedan adquirir las competencias necesarias para afrontar los desafíos con los que inexorablemente se enfrentarán.

La RSU convoca al conjunto de políticas, programas y acciones definidas y ejecutadas por las universidades en beneficio de los actores sociales con los que interactúa y a quienes dirige su acción formadora, de manera que los desarrollos investigativos sobre el tema surgen como una tarea impostergable para mejorar la gestión institucional a fin de cumplir con la función que le fuera asignada por la sociedad.

En el apartado siguiente se comparten dos trabajos de investigación cuyos resultados permiten corroborar algunas de las consideraciones expuestas.

\section{Un aporte desde la investigación}

Con el fin de contribuir a los aportes realizados en materia de RSU en diferentes universidades de América Latina, un equipo de investigadores del Instituto de Responsabilidad Social para el Desarrollo Sustentable - GETACE, de la Universidad Nacional de la Patagonia San Juan Bosco, 
Argentina se propuso incursionar en la temática, por entender la importancia del vínculo directo entre lo que la universidad declara y practica frente a su propia comunidad y a la sociedad en su conjunto.

Así se planteó el desarrollo del proyecto "Responsabilidad social universitaria. Enfoques y prácticas en dos universidades latinoamericanas" (2015-2017), ${ }^{2}$ asociados con un equipo colega, de la Facultad de Administración del Politécnico Colombiano "Jaime Isaza Cadavid" (Medellín, Colombia) y posteriormente su continuidad y complemento, el proyecto "Responsabilidad Social Universitaria: una aproximación a la percepción de los grupos de interés externos" (2018-2020). ${ }^{3}$ En ambos casos, se conformaron unidades ejecutoras ${ }^{4}$ interdisciplinarias con representantes de todos los claustros involucrados.

Se comparten a continuación los lineamientos del proceso investigativo y los resultados obtenidos, sobre el trabajo desarrollado en la institución argentina ${ }^{5}$, a partir del modelo de abordaje diseñado al efecto, que se expone en la figura 1:

Figura 1: Modelo de abordaje de la RSU.

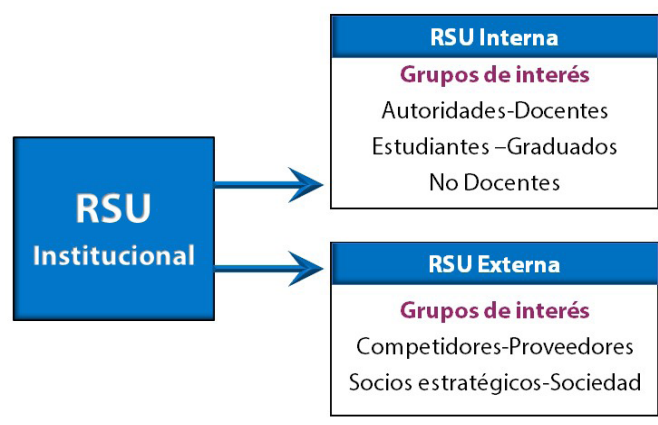

Fuente: Elaboración propia.

${ }^{2}$ PIN $N^{\circ} 1198$ - Res. R/8 N³07-2015 UNPSJB.

${ }^{3} P I N^{\circ} 1397$ - Res. R/9 N495-2018 UNPSBJ.

4 Miembros de las Unidades Ejecutoras: Directora: Dra. Patricia Kent (ex vicedecana), Codirector: Mg. Gabriel Trucco (docente). Docentes: Mg. Araceli Cifuentes Valenzuela y Mg. María Elena Sendín. Graduados: Lic. Denisse Capart, Lic. Luis Salazar, Lic. Rodrigo Domínguez y Lic. Germán Soetbeer. Auxiliares Alumnos Daniel Pugh y Susana Capart. Personal no docente (administrativo): Virginia Echeverría.

${ }^{5}$ El proceso y los resultados de la investigación fueron publicados en: Kent, $P$. et al. (2018). Responsabilidad social universitaria: Una mirada endógena. Revista FACES, 24 (51), 77-91. En la Universidad Nacional de Mar del Plata, Argentina. 
El objetivo de la primera investigación consistió en caracterizar la implementación de enfoques y prácticas de RSU en la unidad académica objeto de estudio desde la mirada de los actores internos, definidos en términos de la normativa institucional vigente, lo cual permitió identificarlos claramente y eliminó la posibilidad de ambigüedades en la conceptualización, quedando integrados por los estamentos con representación en los órganos de decisión institucional: autoridades, docentes, estudiantes, graduados y personal no docente ${ }^{6}$.

Para el abordaje se realizó una adaptación del modelo de Vallaeys (2009, p.12-15) definiendo dos dimensiones de análisis, cada una de las cuales incluía distintos ejes para el relevamiento de información, tal como se muestra en la figura 2 .

Figura 2: Abordaje de la RSU interna. Adaptación del modelo de Vallaeys.

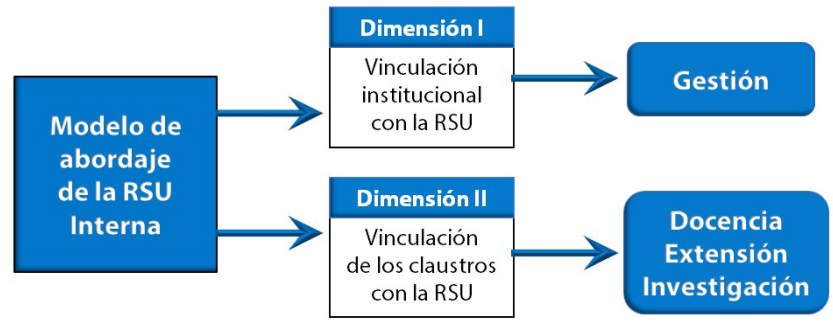

Fuente: Elaboración propia.

Como la investigación universitaria debe procurar una realimentación positiva con el conjunto de la sociedad, en función de la experiencia adquirida y los resultados obtenidos en el primer estudio, que aportó un análisis endógeno, resultaba trascendente conocer la opinión de la totalidad de los actores involucrados, por lo tanto, se planteó una segunda parte con el objetivo de analizar la percepción de los stakeholders (persona, grupo o institución que puede afectar o ser afectada por la organización) externos sobre las prácticas de RSU en la institución.

Atento a que la variedad de los interlocutores de las instituciones de educación superior alcanza al conjunto de la sociedad, se observó que prácticamente la totalidad de actores sociales que operan en su entorno

\footnotetext{
${ }^{6}$ En el contexto de las universidades argentinas se entiende como Personal no docente, al personal de apoyo administrativo, técnico y operativo de los centros universitarios, lo que equivale a personal de administración y servicios en otros países.
} 
directo e indirecto forman parte de los grupos de interés externos, lo cual presentó la dificultad de delimitación de la población objeto de estudio, teniendo en cuenta que:

"en cuanto a la problemática de las "partes interesadas" de la Universidad (sus "stakeholders"), la verdad es que es mucho más difícil designarlas a priori para el ámbito universitario que para otros tipos de organizaciones, puesto que el carácter "universal" de la Universidad hace que casi todos los grupos de interés tengan algo que ver con ella, directa o indirectamente, y puedan sentirse "afectados" e "interesados" en ella, incluso las generaciones futuras, puesto que son ellas que van a sufrir o beneficiarse con la formación que los líderes del mundo habrán recibido en su respectiva casa de estudios". (Vallaeys, 2008, p.212), de modo tal que fue necesario diseñar un modelo ad hoc para el análisis (Figura 3).

Figura 3: Modelo de abordaje de la RSU externa.

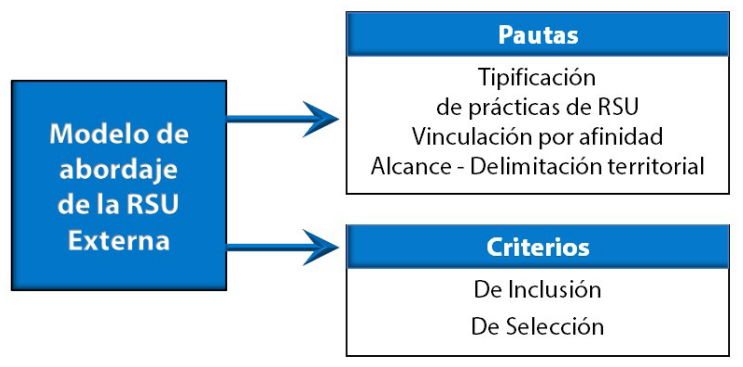

Fuente: Elaboración propia.

Las pautas básicas para la definición del modelo fueron las siguientes:

- Tipificación de las prácticas de RSU: se elaboró un listado de prácticas orientadas a los grupos de interés externos, en función de los antecedentes proporcionados por la investigación precedente.

- Vinculación por afinidad: establecida en función de objetivos comunes, entre los grupos de interés externos y la institución. La identificación hizo necesario establecer parámetros pertinentes a la función que cumple cada uno de ellos.

- Alcance y delimitación territorial: atento al alcance (territorialidad) definido para el proyecto, el trabajo se centró en el relevamiento de los grupos de interés externos del entorno inmediato.

- Definición de criterios de inclusión y de selección:

- Para la inclusión de los actores en un grupo de interés externo, teniendo en cuenta que las personas $u$ organizaciones 
podrían estar incluidas en más de uno, se consideró el rol más significativo de cada una en función de su vínculo con la institución.

- Para la selección de los actores en un grupo de interés externo, se consideró el rol más significativo del mismo en función del alcance del proyecto.

Al considerar la diversidad, heterogeneidad y cantidad de grupos de interés externos identificados en función de los criterios establecidos en el modelo, resultó necesario, adaptando la clasificación expuesta en Vallaeys, (2009, p.11), su agrupamiento en cuatro bloques, definidos de la siguiente manera:

- Competidores: instituciones que "compiten" en ofertas de carreras de grado y/o de posgrado con la institución.

- Proveedores: instituciones que estarían en condiciones de "proveer" demandantes de servicios (alumnos de grado y/o de postgrado) a la institución.

- Socios Estratégicos: organizaciones que "deberían estar vinculadas" con la institución a fin de desarrollar actividades y/o proyectos en forma conjunta.

- Sociedad: personas que "pudieran" tener una opinión sobre las acciones de RSU de la institución. En este caso se seleccionaron dos grupos de actores sociales involucrados, definidos de la siguiente manera:

- Ex - alumnos de grado y de postgrado de la institución: aquellos que por distintos motivos no hubieran completado sus estudios.

- Comunidad: formada por vecinos radicados en localidades situadas en la zona geográfica de influencia inmediata de la institución.

Con una propuesta metodológica similar, ambos estudios fueron caracterizados como investigaciones sociales descriptivas en función de las categorías y unidades de análisis, centradas en la identificación de factores normativos, materiales y simbólicos, que proporcionaron una mirada adecuada a la conceptualización teórica y a la realidad fáctica, sobre las prácticas de RSU institucionales.

Se aplicaron diferentes técnicas cualitativas y cuantitativas, entre ellas: el análisis de material bibliográfico y documental, la observación directa 
participante y no participante, encuestas semiestructuradas, entrevistas de profundidad, y análisis de discurso.

Para el relevamiento de información, a fin de indagar su pertinencia y los impactos institucionales producidos, se trabajó con base en la administración de encuestas virtuales confeccionadas ad-hoc para cada grupo de interés, mediante el diseño de instrumentos compuestos por tres bloques de enunciados empíricos que fueron definidos del siguiente modo:

- $\quad$ El primero orientado a obtener información sobre la vinculación entre la institución y cada grupo de interés externo.

- El segundo, enfocado a la mirada del grupo de interés externo sobre el comportamiento de la institución en términos de su RSU.

- El tercero, conducente a relevar aspectos específicos para cada grupo de interés externo.

Con el fin de viabilizar la tasa de respuesta se aplicó en ambos proyectos la técnica de censo para las autoridades, los docentes, el personal no docente, los competidores y los proveedores; y la técnica de muestreo proporcional estratificado para los graduados, los estudiantes, los socios estratégicos y la sociedad, con el posterior tratamiento estadístico.

La metodología seleccionada permitió identificar no sólo las áreas en las cuales fue posible intervenir para mejorar el cumplimiento de las funciones universitarias, sus actividades cotidianas y sus propuestas, sino también para comparar y analizar las consistencias y/o discrepancias que se observaran en las percepciones manifiestas de los diferentes encuestados.

Cabe señalar como particularidades de estos estudios, que en ambos casos estuvieron orientados a la profundización del diálogo interdisciplinario atento a la disparidad de formación de base de los investigadores participantes:

- Se trabajó con una metodología participativa que enriqueció el proceso, sus resultados y sus conclusiones.

- Ambos proyectos representan el primer antecedente de abordaje de la RSU, a partir de la mirada de sus grupos de interés, en la institución objeto de estudio.

- Por primera vez un miembro del claustro no docente integró una unidad ejecutora de un proyecto de investigación en la institución, aportando una mirada que no podía estar ausente.

- Formaron parte del equipo dos profesionales no egresados de la institución y sin vinculación con la misma, lo que brindó una mayor objetividad al análisis. 
Las conclusiones más significativas sobre los resultados obtenidos del relevamiento a los grupos de interés internos fueron las siguientes:

- Existe una significativa inconsistencia en las bases de datos de contactos de la institución.

- Se revelan coincidencias en las respuestas de todos los claustros en cuanto a la necesidad de implementación de un código de ética institucional, a la no percepción de situaciones de discriminación de ningún tipo y a la necesidad de adaptación de la infraestructura edilicia para personas con capacidades diferentes.

- La mitad de los encuestados de todos los claustros, excepto las autoridades, aseguran no recibir regularmente información sobre actividades académicas, de investigación y de extensión.

- Todos los claustros, excepto las autoridades, manifiestan la necesidad de promoción y capacitación en temas vinculados a responsabilidad social y a proyectos sociales relacionados con la región.

- Con respecto a la RSU institucional los docentes muestran una mirada más rigurosa que la de los estudiantes, que la expuesta por el claustro no docente y que la revelada por las autoridades; siendo más indulgentes en comparación con la expresada por los graduados.

- La mitad de los estudiantes no incluye al programa de voluntariado y a las actividades de responsabilidad social como parte de la vida universitaria.

- Los graduados manifiestan su distanciamiento, desvinculación y desconocimiento sobre la gestión, asimismo, aseguran mantener una interacción distante y discontinua a partir de vínculos de comunicación esporádicos con la institución, expresando que no participan de la vida universitaria.

- Seobserva una notoria disparidad en la percepción delas autoridades sobre el desarrollo de prácticas de RSU con relación al resto de los estamentos. Se destaca particularmente el aspecto referido a las comunicaciones impulsadas desde la institución, que no resultan eficientes en opinión de los claustros, mientras que las autoridades consideran que el proceso comunicacional es adecuado.

Con respecto a los resultados obtenidos en la segunda investigación, en la tabla 1 se exponen las respuestas de los grupos de interés externos (en \%) sobre los enunciados propuestos, en términos de acuerdo (A), desacuerdo (D) o desconocimiento $(\mathrm{N})$ con la difusión o promoción de actividades y/o proyectos institucionales: 
Tabla 1: Resultados relevamiento a GIE externos.

\begin{tabular}{|c|c|c|c|c|c|c|c|c|c|c|c|c|c|c|c|}
\hline \multirow[t]{2}{*}{ Enunciados } & \multicolumn{3}{|c|}{ Competidores } & \multicolumn{3}{|c|}{ Proveedores } & \multicolumn{3}{|c|}{ Socios E. } & \multicolumn{3}{|c|}{ Comunidad } & \multicolumn{3}{|c|}{ Exalumnos } \\
\hline & A & $D$ & $\mathrm{~N}$ & A & D & $N$ & A & $D$ & $N$ & $A$ & D & $\mathrm{N}$ & A & $D$ & $\mathrm{~N}$ \\
\hline Oferta académica & 100 & 0 & 0 & 100 & 0 & 0 & 89 & 4 & 7 & 87 & 2 & 11 & 88 & 7 & 5 \\
\hline $\begin{array}{l}\text { Campo ocupacional } \\
\text { de egresados }\end{array}$ & 50 & 25 & 25 & 50 & 0 & 50 & 32 & 14 & 54 & 43 & 25 & 32 & 51 & 26 & 23 \\
\hline Pasantes & 50 & 25 & 25 & 50 & 0 & 50 & 46 & 25 & 29 & 39 & 29 & 32 & 40 & 33 & 27 \\
\hline Investigación & 75 & 25 & 0 & 33 & 0 & 67 & 50 & 14 & 36 & 41 & 31 & 28 & 43 & 28 & 29 \\
\hline Extensión & 100 & 0 & 0 & 50 & 0 & 50 & 61 & 14 & 25 & 50 & 25 & 25 & 46 & 33 & 21 \\
\hline $\begin{array}{l}\text { Problemáticas } \\
\text { Socio-ambientales }\end{array}$ & 50 & 25 & 25 & 50 & 0 & 50 & 46 & 11 & 43 & 52 & 20 & 28 & 41 & 26 & 33 \\
\hline $\begin{array}{l}\text { Programa } \\
\text { de Voluntariado }\end{array}$ & 50 & 25 & 25 & 50 & 0 & 50 & 21 & 18 & 61 & 38 & 23 & 39 & 34 & 26 & 40 \\
\hline Hábitos sustentables & 50 & 25 & 25 & 33 & 0 & 67 & 36 & 21 & 43 & 26 & 35 & 39 & 38 & 27 & 35 \\
\hline $\begin{array}{l}\text { Logros } \\
\text { de sus miembros }\end{array}$ & 25 & 25 & 50 & 33 & 0 & 67 & 39 & 18 & 43 & 37 & 35 & 28 & 43 & 29 & 28 \\
\hline $\begin{array}{l}\text { Actividades } \\
\text { de agrupaciones }\end{array}$ & 50 & 25 & 25 & 50 & 0 & 50 & 39 & 11 & 50 & 35 & 20 & 45 & 47 & 22 & 31 \\
\hline
\end{tabular}

Fuente: Elaboración propia.

En la figura 4 se muestran los resultados sobre la calificación asignada a la RSU de la institución por los grupos de interés encuestados en ambos proyectos, en una escala del 1 al 10, donde 10 corresponde a la mejor puntuación.

Figura 4: Percepción de los grupos de interés sobre la RSU institucional



$\square$ RSU

Fuente: Elaboración propia.

Como corolario de las investigaciones desarrolladas se observa en general para los grupos de interés internos y externos que: 
- Los encuestados denotan cierta inquietud e interés, así como también un significativo desconocimiento general por la temática. Es posible que estos indicadores se originen en la incipiente incorporación de la responsabilidad social en los distintos ámbitos tanto internos como externos.

- Se obtuvo un bajo nivel de respuestas, a pesar de las reiteraciones en la solicitud, en particular en el caso de los grupos de interés externos que, en alguna medida, pudo tener su causa en lo que se conoce como "fatiga de encuestas", dada la gran cantidad de requerimientos de este tipo en la actualidad. Esta situación fue advertida como probable inicialmente por las unidades ejecutoras, no obstante, se optó por la metodología descrita en función de sus beneficios relacionados con la simplicidad y factibilidad de abordaje.

- En ambos proyectos se pone de manifiesto el exiguo compromiso social de la institución reflejado en su escasa atención a las demandas y expectativas de sus grupos de interés internos y externos.

"Al producir conocimientos fruto de la investigación las universidades no sólo educan, además promueven la innovación fundamental para lograr un crecimiento armónico. Sus impactos indudablemente deberán propender a lograr una realimentación positiva con la sociedad teniendo en cuenta diversos aspectos académicos, humanos, éticos, productivos e institucionales". (Kent, 2018, p. 78)

\section{De la reflexión a la acción}

La RSU como camino hacia el desarrollo humano sostenible, debe convertirse en una alternativa de cambio para la gestión universitaria que se alcanzará transitando un proceso complejo que facilite nuevas opciones en el ejercicio de sus funciones constitutivas imbuidas de esta mirada.

Se desprende de la revisión bibliográfica sobre el tema, que existe hoy en las instituciones de educación superior una mayor conciencia sobre su responsabilidad social a partir de la reflexión y de la observación de los resultados de algunas iniciativas de probado éxito, pero es preciso generalizar, sostener y mejorar esas prácticas de manera permanente, en concordancia con los complejos retos sociales de la actualidad.

Como se observa en el caso expuesto en el apartado anterior, los grupos de interés participantes de la vida universitaria, en particular sus claustros, deben conocer y apropiarse de los contenidos de la RSU y de sus consiguientes ventajas, en términos de la relevante función social que están llamados a cumplir. 




Atender las opiniones y requerimientos de los stakeholders con los que interactúa y a quienes se debe, requiere que la universidad modifique los sistemas y mecanismos de comunicación, diseñe e implemente planes, programas y proyectos que tiendan a profundizar los conceptos de RSU, y revise los procedimientos de gestión para que efectivamente promuevan la transparencia y congruencia con la misión y la visión sostenidas en las declaraciones institucionales.

Las instituciones de educación superior deben incorporar el paradigma de la RSU en su cultura organizacional para ofrecer a la sociedad graduados capaces de brindar respuestas a sus demandas.

Abordar este tema hoy amerita su contextualización en el marco de la pandemia que afecta al planeta y que está provocando una significativa reconversión en la vida de los ciudadanos del mundo, y obviamente también en la vida universitaria.

El Covid19 interpela a la humanidad con situaciones en las cuales el espacio reservado a la RSU resultará clave porque la sociedad postpandemia será muy distinta. Cambiaron los hábitos, las formas de trabajo, de interacción, de modo tal que será preciso reconocer y atender no solo el evidente impacto económico, sino también las consecuencias sociales y ambientales que conlleva este nuevo escenario, lo cual induce a repensar modos de conducción diferentes, sostenidos en posturas éticas con altos niveles de sensibilidad social.

El rol de la universidad como factor de cambio social y ambiental, implica redefinir un nuevo liderazgo, solidario y colaborativo, para contribuir a reconstruir confianza y a planificar un futuro sostenible postCovid19, con igualdad de oportunidades de acceso y permanencia a una educación superior solidaria, diseñando e implementando estrategias integrales, equitativas e inclusivas contando con la cooperación de los grupos de interés internos y externos.

El sistema universitario enfrenta un desafío sin precedentes que le impone aprender a partir de la crisis para gestionar las instituciones, identificando caminos que conduzcan a una nueva sostenibilidad con responsabilidad social.

Llegó el tiempo de pasar a la acción. La sociedad reclama a las universidades asumir sus compromisos sociales y ambientales; existen mecanismos para reconvertirlas en instituciones socialmente responsables, solo resta, con convicción y formación, tomar la decisión e iniciar el recorrido. 
Para finalizar, se comparte la suscripción de un nuevo contrato social entre la universidad y la sociedad, propuesto por Vallaeys $(2011$, p.18) en los siguientes términos:

"Tú, Sociedad, me garantizas autonomía y recursos, y yo, Universidad, te doy:

1) más Democracia, a través de la formación de estudiantes y ciudadanos responsables;

2) más Ciencia responsable, lúcida y abierta a la solución de los problemas sociales de la humanidad, y

3) mejor Desarrollo equitativo, innovador y sostenible, con profesionales competentes y comprometidos".

¿Qué diría la gente, recortada y vacía, si en un día fortuito, por ultrafantasía, me tiñera el cabello de plateado y violeta, usara peplo griego, cambiara la peineta por cintillo de flores: miosotis o jazmines, cantara por las calles al compás de violines, o dijera mis versos recorriendo las plazas, libertado mi gusto de vulgares mordazas? ¿Irían a mirarme cubriendo las aceras? ¿Me quemarían como quemaron hechiceras? ¿Campanas tocarían para llamar a misa? En verdad que pensarlo me da un poco de risa.

Alfonsina Storni Argentina.

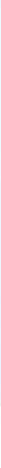




\section{Referencias bibliográficas}

1. Calderón, I. (2016). Responsabilidad social universitaria; una acción impostergable. En: Izarra Vielma, D. Experiencias y perspectivas en ética profesional y responsabilidad social universitaria en Iberoamérica. Caracas: Universidad Pedagógica Experimental Libertador. Pp. 191-208.

2. Chile. Proyecto Universidad: Construye País. (2001-2006) Observando la responsabilidad social universitaria. Corporación Participa y Fundación Avina. Disponible en Internet: http://www.construyepais.cl / http://www.cyta.com.ar/ biblioteca/bddoc/bdlibros/rse/334_as_observando_la_rsu.pdf.

3. Cleri, C. (2007). El libro de las pymes. 1ª. ed. Buenos Aires: Granica. 448 pp.

4. Kent, P. et al. (2018). Responsabilidad social universitaria: Una mirada endógena. Revista FACES, 24 (51), 77-91. Mar del Plata (Arg.): Universidad Nacional de Mar del Plata.

5. Naciones Unidas (2015). 17 objetivos para transformar al mundo. Disponible en Internet: https://www.un.org/sustainabledevelopment/es/

6. Schvarstein, L. (2003). La inteligencia social de las organizaciones. Desarrollando las competencias necesarias para el ejercicio efectivo de la responsabilidad social. $1^{\text {a }}$ ed. Buenos Aires: Paidós. 272 Pp.

7. Torres P., M. y Trápaga O., M. (2012). Una aproximación teórica al concepto de Responsabilidad Social Universitaria. En: Pérez C., M. et al. Responsabilidad Social de las Universidades. Tomo IV. Buenos Aires: Red Latinoamericana de Cooperación Universitaria. Pp. 175-189.

8. Vallaeys, F. (2008). Responsabilidad social universitaria. una nueva filosofía de gestión ética e inteligente para las universidades. Educación Superior y Sociedad,13, (2), 191220. http://www.iesalc.unesco.org/ess/index.php/ess3/issue/view/37/38

9. Vallaeys, F. (2011) ¿Qué es la Responsabilidad Social Universitaria? Disponible en Internet: http://voluntariadofce.econ.uba.ar/wp-content/uploads/2011/08/Que-esla-RSU.-Vallaeys.pdf

10. Vallaeys, F.; De la Cruz, C. y Sasia, P. M. (2009). Responsabilidad social universitaria. Manual de primeros pasos. $1^{\circ}$ ed. Ciudad de Méjico: BID. Mc Graw Hill. 85 pp.

Para citar este artículo:
Kent, P. (2020). Universidades socialmente responsables. Teuken Bidikay 11 (17), 131-148. doi: 10.33571/teuken.v11n17a7

Ge: HJS.

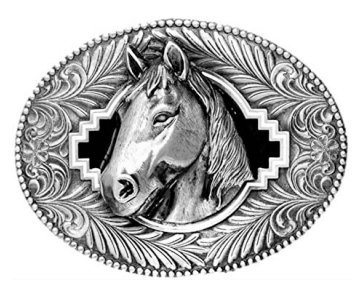

